\title{
Performance Analysis of an Insert Cooling System for Long-Pulse Operation of a Coaxial-Cavity Gyrotron
}

\author{
Parth C Kalaria ${ }^{1}$, Marc George ${ }^{3,4}$, Stefan Illy ${ }^{1}$, Konstantinos A Avramidis ${ }^{1}$, \\ Gerd Gantenbein ${ }^{1}$, Sebastian Ruess ${ }^{1,2}$, Tomasz Rzesnicki ${ }^{1}$, \\ Manfred Thumm ${ }^{1,2}$ and John Jelonnek ${ }^{1,2}$ \\ ${ }^{1}$ Institute for Pulsed Power and Microwave Technology (IHM), \\ ${ }^{2}$ Institute of Radio Frequency Engineering and Electronics (IHE), \\ Karlsruhe Institute of Technology (KIT), Karlsruhe, Germany. \\ ${ }^{3}$ Ecole Centrale de Marseille, Marseille, France. \\ ${ }^{4}$ Aix-Marseille Université, Marseille, France.
}

\begin{abstract}
The successful operation of a $2 \mathrm{MW} 170 \mathrm{GHz}$ coaxial-cavity gyrotron has been demonstrated at IHM-KIT in short(ms) pulses and the ongoing research activities are aiming to upgrade the existing tube to the long-pulse regime. In this work, the performance of the insert cooling system is systematically studied for longpulse coaxial-cavity gyrotron operation. A multi-physics simulation model is developed with the relevant turbulence model and mesh parameters. The results suggest stable operation of the insert under continuous wave (CW) operating condition. In the case of perfectly aligned insert, the maximum temperature of the cooling water is below the boiling temperature and the deformations of the insert geometry are significantly small enough not to influence the performance of the gyrotron. Additionally, the operational limits of the insert cooling system are determined with respect to the maximum allowable heatflux.
\end{abstract}

Keywords: Nuclear fusion; Electron Cyclotron Resonance Heating and Current Drive (ECRH\&CD); gyrotron; coaxial cavity; insert loading analysis; insert misalignment; thermo-hydraulic simulations; thermomechanical simulations

\section{Introduction}

High-frequency $(100 \mathrm{GHz}-300 \mathrm{GHz})$, high-power $(\sim 1-$ $2 \mathrm{MW}$ ) gyrotrons are the prominent RF sources in the thermonuclear fusion experiments for various applications like: Electron Cyclotron Resonance Heating (ECRH), noninductive Current Drive (CD), Collective Thomson Scattering (CTS) and plasma stability control [1]. For the ITER tokamak in Cadarache (France), twenty-four $170 \mathrm{GHz}$ gyrotrons are planned for a total ECRH\&CD power requirement of $20 \mathrm{MW}$. In Europe, a $1 \mathrm{MW}$ $170 \mathrm{GHz}$ hollow-cavity gyrotron and a $2 \mathrm{MW} 170 \mathrm{GHz}$ coaxial-cavity gyrotron have been developed for ITER [2]. Compared to the hollow-cavity design, the corrugatedinsert in a coaxial gyrotron cavity significantly controls the mode competition and allow high-power gyrotron operation with very high-order operating mode. During the gyrotron operation, the cavity surface and the coaxial insert are heated by the generated RF wave. The increase in temperature results in deformations of cavity wall and insert, which further modifies the operating conditions, the generated RF waves, and the total heat flux. The effectiveness of various cooling systems for the cavity outer wall has been studied systematically in [3-7]. In the present work, the performance of the existing insert cooling system is numerically verified for $\mathrm{CW}$ operation of a $2 \mathrm{MW} 170 \mathrm{GHz}$ coaxial-cavity gyrotron [8].

\section{Simulation Set-up}

The simple schematic of the insert cooling system is presented in Figure 1. The water flows to the top of the insert in a central inlet pipe, then flows back between the pipe's outer wall and the inner inlet wall. With the nominal operating condition, it had to be checked that the insert would be able to withstand $\mathrm{CW}$ operation without significant deformation. The overview of the adopted simulation approach is presented in Figure 2 and it is similar to that used in [3] [7]. First, with help of the electromagnetic (EM) simulation, the heat flux corresponding to the original geometry is calculated at room temperature. The calculated heat flux is imposed on the insert wall to obtain the temperature field profile in the thermal-hydraulic (TH) simulation. In the next step, the deformations of the structure are calculated in thermomechanical (TM) simulation using the new temperature profile. The deformed geometry and new temperature profile are then used for the calculation of the new heat flux in EM simulation.

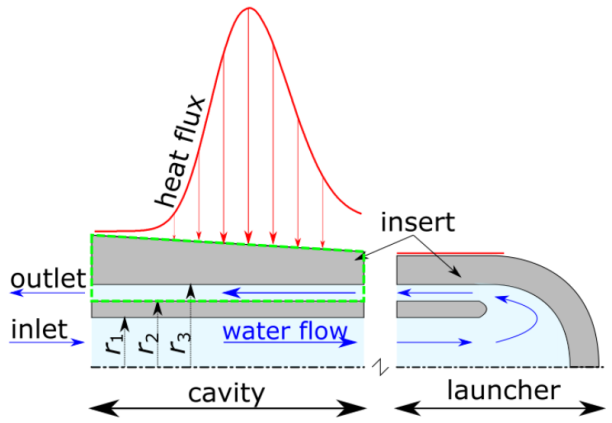

Figure 1. A simple sketch of the existing insert cooling system with the heat flux profile. The values of $r_{1}, r_{2}$ and $r_{3}$ are $3 \mathrm{~mm}, 4 \mathrm{~mm}$ and $5 \mathrm{~mm}$, respectively. The selected sector for the multi-physics simulations is highlighted with green dashed border. 
Table 1. Important simulation results in the case of the perfectly aligned insert under nominal operating conditions. (The deformations are given relative to the original geometric configuration.)

\begin{tabular}{|c|c|c|c|c|c|}
\hline simulation & & iteration 0 & iteration 1 & iteration 2 & iteration 3 \\
\hline Electromagnetic (EM) & maximum heat flux at wall $\left(\mathrm{kW} / \mathrm{cm}^{2}\right)$ & 0.113 & 0.120 & 0.121 & 0.121 \\
\hline \multirow{2}{*}{$\begin{array}{l}\text { Thermal-Hydraulic } \\
\text { (TH) }\end{array}$} & maximum temperature in GlidCop $\left({ }^{\circ} \mathrm{C}\right)$ & 70.8 & 73.5 & 73.7 & \\
\hline & maximum temperature in water $\left({ }^{\circ} \mathrm{C}\right)$ & 62.8 & 65.0 & 65.1 & \\
\hline \multirow{3}{*}{ Thermo-Mechanical (TM) } & maximum equivalent stress $(\mathrm{MPa})$ & 44 & 47 & 47 & \\
\hline & maximum radial deformation $(\mu \mathrm{m})$ & 5.3 & 5.6 & 5.6 & \\
\hline & maximum axial deformation $(\mu \mathrm{m})$ & 17.3 & 18.3 & 18.4 & \\
\hline
\end{tabular}

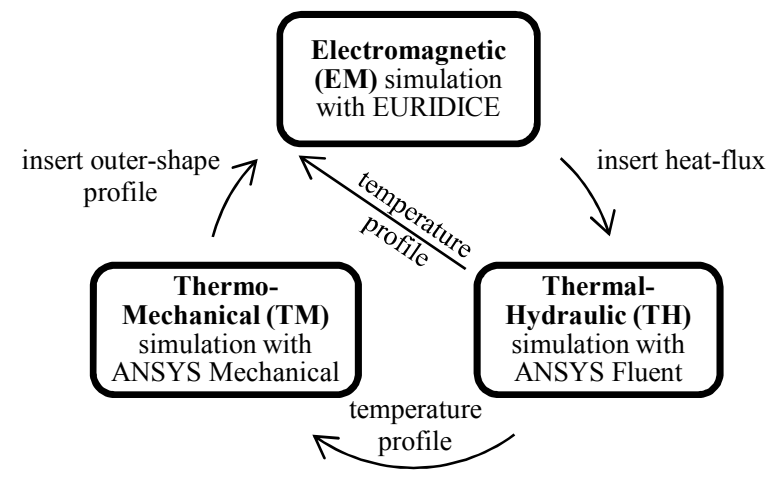

Figure 2. Overview of the selected iterative simulation approach.

As the value of the insert heat flux is negligible outside the cavity region, only the cavity region is considered in the simulations to minimize computational resources. Considering the cylindrical symmetry of the aligned insert, a $10^{\circ}$-degree slice of the insert is selected for the analysis using rotationally periodic boundary conditions. The maximum mesh cell size of $0.1 \mathrm{~mm}$ is selected in the axial direction for the solid and fluid region. At the inlet of the fluid domain, a flow speed of $5.9 \mathrm{~m} / \mathrm{s}$ are imposed, which for the adopted dimensions corresponds to the realistic flow rate of $10 \mathrm{~L} / \mathrm{min}$. This flow rate supports the operation of the insert without significant vibration with a reasonable security margin. The SST (Shear-Stress Transport) k- $\omega$ turbulence model is adopted for the TH simulation.

\section{Results}

The results of the simulations considering the nominal operating condition and a perfectly aligned GlidCop Al-15 insert are summarized in Table-I. For the iteration 0, uniform temperature of $27^{\circ} \mathrm{C}$ is selected with the standard non-deformed geometries. The convergence condition of this iterative simulations is satisfied when the new heat flux calculated by EURIDICE is not more than $0.2 \%$ different from that used in the previous step. The maximum temperature of the water is well below the boiling temperature of $160^{\circ} \mathrm{C}$ (boiling temperature at the given water pressure of 6.2 bar). The deformations of the insert geometry are very small which does not alter the performance of the gyrotron. After successfully validating the existing insert cooling system for long-pulse gyrotron operation, the maximum acceptable value for the insert loading was determined. Two ways were selected to increase the heat flux: (a) by increasing the electron beam current and consequently the generated microwave power without changing the geometry of the insert and (b) by increasing the radius of the insert, thus making this closer to the field maximum, without changing the electron beam current. The results indicate stable insert operation up to insert loading of $0.39 \mathrm{~kW} / \mathrm{cm}^{2}$. This value is almost two times to the considered insert loading limit of $0.2 \mathrm{~kW} / \mathrm{cm}^{2}$, usually assumed during gyrotron design.

\section{Acknowledgements}

This work has been carried out within the framework of the EUROfusion Consortium and has received funding from the Euratom research and training programme 2014-2018 under grant agreement No 633053. The views and opinions expressed herein do not necessarily reflect those of the European Commission. Fruitful discussions with Andrea Bertinetti are kindly acknowledged.

\section{References}

1. Thumm M. 2017 State-of-the-art of high power gyrodevices and free electron masers, update 2016 Sci. Rep. KIT-SR 7735 (DOI: 10.5445/KSP/1000068193)

2. Jelonnek J. et al 2017 Fusion Engineering and Design 123 241-46

3. Bertinetti A. et al 2017 Fusion Engineering and Design 123 313-16

4. Savoldi L. et al 2016 IEEE Trans. on Plasma Science, 64 3885-92

5. Savoldi L. et al 2015 IEEE Symposium on Fusion Engineering (Austin, TX, USA), (DOI: 10.1109/ SOFE.2015.7482420)

6. Avramidis K. A. et al 2017 IEEE International Vacuum Electronics Conference (London, UK)

7. Avramidis K. A. et al 2018 IEEE Trans. Electron Devices, accepted for publication.

8. Ruess S. et al $47^{\text {th }}$ European Microwave Conference, 8 - 12 October 2017, Nuremberg, Germany.

9. Avramides K. A. et al $201217^{\text {th }}$ Joint Workshop on Electron Cyclotron Emission and Electron Cyclotron Resonance Heating (Deurne, The Netherlands) (DOI: 10.1051/epjconf/20123204016) 